\title{
A Framework and Model for Estimating the Individual Benefits and Costs of Exercise for Long Run Health
}

\author{
Michael D. Everett ${ }^{* 1}$ and Michael W. Ramsey ${ }^{2}$ \\ ${ }^{I}$ Department of Economics and Finance, College of Business and Technology, East Tennessee State University, Johnson \\ City, TN 37614, USA \\ ${ }^{2}$ Department of Kinesiology, Leisure \& Sport Sciences, College of Education, East Tennessee State University, Johnson \\ City, TN 37614, USA
}

\begin{abstract}
This paper develops a computer spreadsheet framework and model for estimating the individual benefits and costs of exercise for long run health. The biological costs and benefits rest on solid production functions between exercise intensity levels, fitness levels, and two key indices of long run health outputs - probability of all cause mortality and an index of health care costs. The less certain monetary estimates of those costs and benefits come from reviews of the key literature. The resulting model of individual exercise costs and benefits provides a basis for individual long run health planning plus cost effective and cost benefit analysis of different exercise strategies. The latter, which uses the implicit value of life, may provide insights to the lack of consistent exercise and strategies to increase it.
\end{abstract}

Keywords: Cost benefit of exercise, exercise costs, exercise financial benefits, fitness and health.

\section{INTRODUCTION}

Over the last decade several large biological studies have related five objectively measured fitness levels to substantial reductions in the relative risk of all cause mortality (ACM) for middle aged individuals during follow-up periods [1]. A recent study reworked the relationships from relative risk to absolute probability of ACM for different fitness levels [2] and the threshold exercise intensities needed to develop those different fitness levels. Another recent study related health care costs (HCC) to the same five fitness levels for a similar age group [3]. These relationships provide a solid biological basis for estimating the long run benefits and costs of aerobic exercise.

This paper builds on those relationships, or production functions as economists call them, to develop a computer spreadsheet framework and model for estimating the individual monetary costs and benefits of the exercise inputs and expected longrun health outputs. Such a framework and model could help individuals and their advisors engage in more rigorous long-run health planning and also develop more systematic financial and utility analyses of different exercise strategies in attempts to find optimal ones. The framework and model may also help improve social benefit cost frameworks for analyzing exercise programs and facilities, such as walk and bikeways.

Benefit cost models of individual and household production have provided useful insights into a wide range of other human behaviors from fertility to crime [4]. Although several broad social benefit cost studies of exercise facilities and programs exist [5-11] and some studies have applied general economic analysis to the lack of exercise and strategies to increase it [12-15], no quantitative models of individual or

\footnotetext{
*Address correspondence to this author at the Department of Economics and Finance, College of Business and Technology, East Tennessee State University, Johnson City, TN 37614, USA; E-mail: everettm@etsu.edu
}

household exercise benefits and costs emerge from broad literature searches. Rather, psychological models of exercise behavior have dominated the exercise literature $[16,17]$. However, these models do not systematically include economic variables, such as the opportunity cost of exercise time and the discounted monetary value of long-run expected health benefits.

After a description of the methodology, the article presents the framework and model in a demonstration and explanation section starting with an easy-to-use data input table. More casual users can go directly to the net benefits in the data output tables. That section also explains how the framework and model derive those net benefits in detail for the interested reader and allows further changes in model inputs. A discussion section explores the implications of the model and evaluates the reliability and limitations of the model parameters.

\section{METHODOLOGY}

This individual exercise benefit cost framework and model uses biological and economic methodologies which are accessible to a wide range of individuals from different backgrounds. First, the framework uses a widely available computer spreadsheet program (Excel) with a user data input table and various output tables with clear bottom line costs, benefits, and net benefits to exercise. The framework becomes a model of exercise benefits and costs with assumptions about values for the key parameters. The model in this paper utilizes recently developed relatively precise and clear biological production functions between exercise, fitness, and absolute probabilities of ACM and $\mathrm{HCC}[2,3]$. The estimates of the monetary costs and benefits of exercise and increased long-run health rest on standard economic and financial techniques. The basic rational economic model of human behavior assumes individuals attempt to maximize utility or well-being by undertaking those activities for 
which the expected increase in benefits exceed the expected increase in costs. These include both explicit (obvious or tangible) and implicit (foregone opportunities). People usually value future costs and benefits less than present ones. Thus, the model uses basic financial formulas for discounting future benefits and costs back to present values. Estimates of these variables, such as time costs, the value of a life, and discount rates, come from a broad review of the literature using Googlescholar.

The underlying biomedical studies require the framework and model to make abstract or stylized assumptions about the exercise training period and a follow-up health benefit period. The standard assumption, which can be relaxed, assumes a 45-year-old male is considering a ten year exercise program to achieve and/or maintain a given level of fitness before a ten year follow-up benefit period which starts at age 55. Higher levels of fitness at the beginning of the follow-up period correlate with lower probabilities of ACM and a lower HCC index. Also, the framework in this paper does not count the costs of any exercise during the follow-up period against the benefits in that period. That exercise would generate benefits in another, later ten year follow-up period.

These stylized assumptions are necessary because of the underlying biological production functions. The large population studies underlying the fitness-ACM production function just measured the fitness of middle-aged individuals averaging around 55-years-old but ranging from their 30 s to their 70s. Then the studies followed the death registers for approximately ten years [18-22]. The HCC study measured the fitness levels for men and women with an average age of 59 years and followed their HCC cost records for one year. Thus, although biological and economic benefits to exercise and fitness undoubtedly occurred before the follow-up period, precise measures for the drop in the probability of ACM and HCC exist only for the follow-up period.

\section{DEMONSTRATION AND EXPLANATION OF THE FRAMEWORK AND MODEL}

This section of the paper demonstrates and explains the framework and model for both the non-technical and technical users. The non-technical user can input their data into Table 1 and then go to the output data which usually fall at the bottom of the output tables. This section also provides an explanation of how each table generates its results for the technical user.

\section{The User Data Input Table}

Note the following data inputs in Table 1 represent just basic data which most users can estimate from their personal situations or can make judgments about from a brief description of the literature. More advanced users can go into the output tables and make additional changes. Also note that throughout the model, shaded cells indicate individualized data which the user has entered. The non-shaded cells contain calculations which the framework makes or assumptions about key parameters from the literature necessary to convert the framework into a model.

In the basic user data input (Table 1), the user first must enter data for output (Table 2) which contains production functions between fitness, health, and exercise. These data require estimates of the exercise time per session for differ- ent possible exercise programs. The sessions per week come from the literature. These programs have different intensities necessary to produce or maintain different peak fitness levels which the top of the table lists. The exercise time per session depends partly on the exercise-fitness production functions such as figure 2 in [2] and across the top rows of Table $\mathbf{1}$ and in the middle of Table 2 . The basic exercise prescriptions call for approximately 30 minutes of moderate exercise five days per week. However, Tables $\mathbf{1}$ and $\mathbf{2}$ show the full range of exercise threshold intensities needed to increase fitness across five levels and maintain that fitness to age limited maxima. As the tables indicate, a very low-fit individual who engages in "light" exercise seven times a week can achieve a low-fit level. Then they would need to increase their intensity to "moderate" five times per week to achieve mid-fit levels. Higher levels of fitness require higher intensities and probably much higher effort, but can occur with only three or four sessions per week. The example in Table 1 assumes many individuals can perform 30 minutes of light and moderate exercise walking near home without changing clothes. "Vigorous" exercise usually requires clothes changing and perhaps some travel to an exercise location. "Hard" intensity exercise may require more extensive travel for engaging in social sports which can provide needed stimulus to work at 90 percent of maximum heart rate. Finally, users must put in the "assumed years of training before the follow-up period" starts and the long-run health benefits of the training begin around age 55 to 65 . This example assumes the standard 10 years for middle-aged males.

Model users can change these exercise time assumptions to fit their personal situations. For example, younger users would need to assume longer training periods before the follow-up benefit period. Also, some individuals may be able to obtain their exercise as a low time-cost joint product with more immediately desired outputs such as transportation. Walking or bicycling for short trips particularly in congested areas where motor vehicle travel remains slow provide examples. Several studies indicate that individuals can break moderate exercise into three ten minute segments per day [23-25], such as walking from their cars to their offices or other destinations. For higher intensity exercise, individuals could use sprint interval training in which they exert maximum effort for 30 seconds and then take up to 4.5 minutes of low effort rest six times in a 30 minute session [26-29]. If the rest intervals could be used for other valued purposes, such as reading on a stationary bike stand or watching the news on television, the exercise time would only take 3 minutes. In general, however, most individuals probably would need at least 30 minutes to an hour to prepare for and engage in a moderate to higher level exercise session.

To estimate the monetary value of the exercise time costs for Table 3 the user must first input an assumed dollar value per hour for time spent exercising in the next section of Table 1. The literature tends to use the average wage rate for busy working days such, as studies on commuting to work, and half that for leisure time, such as non-working days or hours during the day $[30,31]$. For a more accurate monetary estimate of time costs, users or their advisors might ask how much money he or she would need to stop current activities and devote an hour to exercise. The user should assume the exercise would require no effort or impose no other costs and would generate no short or long-run health benefits. 
Table 1. User Data Input Table

For Table 2: Production Functions Between Fitness, Health, and Exercise

\begin{tabular}{|c|c|c|c|c|c|c|}
\hline & & \multirow{2}{*}{\multicolumn{5}{|c|}{ Peak Fitness Levels (in METs*) }} \\
\hline & \multirow[b]{2}{*}{ Description } & & & & & \\
\hline & & Very Low & Low & Mid & High & Very High \\
\hline & METS* & $(<6)$ & $(6-8)$ & $(8-10)$ & $(10-12)$ & $(>12)$ \\
\hline \multicolumn{2}{|l|}{ Threshold exercise intensities of $\mathbf{3 0}$ minute sessions to achieve different fitness levels: } & & & & & \\
\hline \multicolumn{2}{|l|}{ Description of threshold exercise intensity to reach each fitness level } & Sedentary & Light & Moderate & Vigorous & Hard \\
\hline \multicolumn{2}{|l|}{ Approximate percent of age adjusted maximum heart rate (HR) (220 minus age) } & $50 \%$ & $60 \%$ & $70 \%$ & $80 \%$ & $90 \%$ \\
\hline \multicolumn{7}{|l|}{ Time requirements for exercise inputs including travel and clothes changing time } \\
\hline \multicolumn{2}{|l|}{ Hours per session } & 0 & 0.5 & 0.75 & 1 & 1.75 \\
\hline \multicolumn{2}{|l|}{ Sessions per week } & 0 & 7 & 5 & 4 & 3 \\
\hline Assumed years of training before the follow-up period** & 10 & & & & & \\
\hline
\end{tabular}

**Do not include continued training during the follow-up period. Such training would generate health benefits in another follow up period.

For Table 3: The Costs of Exercise Inputs Needed to Increase Fitness Levels

\begin{tabular}{|c|c|c|c|c|c|c|}
\hline Assumed value of time per hour* & $\$ 30$ & & & & & \\
\hline \multicolumn{2}{|l|}{ Other per year exercise costs such as purchased inputs and effort } & & & & & \\
\hline \multicolumn{2}{|l|}{ Purchased inputs on a yearly basis such as gym memberships } & $\$ 0$ & $\$ 0$ & $\$ 0$ & $\$ 0$ & $\$ 0$ \\
\hline \multicolumn{2}{|l|}{ Effort costs per hour of exercise for each exercise level } & $\$ 0$ & $\$ 0$ & $\$ 0$ & $\$ 0$ & $\$ 0$ \\
\hline \multicolumn{2}{|l|}{ Other yearly exercise costs or benefits (enter capital savings as a negative cost) ${ }^{* *}$} & $\$ 0$ & $\$ 0$ & $\$ 0$ & $\$ 0$ & $\$ 0$ \\
\hline Exercise cost discount rate & $3.0 \%$ & & & & & \\
\hline
\end{tabular}

${ }^{*}$ Could range from zero through average wage rate to high marginal value (e.g., what individual would need to stop present activity for an hour).

**These include operating and capital ownership savings when a person engages in a joint product exercise strategy using labor intensive technology such as walking or bicycling to work instead of driving and perhaps owning another household car.

\section{For Table 4: The Explicit Monetary Benefits of Increased Fitness Levels}

\begin{tabular}{|l|c|}
\hline Explicit monetary benefits of reduced ACM probabilities during follow-up & $\$ 80,000$ \\
\hline Expected yearly income during the follow-up period & $\$ 50,000$ \\
\hline Less yearly life insurance payout or survivor annuity if ACM occurs & \\
\hline Monetary benefits of reduced health care costs (HCC) during follow-up period & $\$ 1,000$ \\
\hline Expected yearly HCC after insurance for a mid-fit person & Discount rate for those explicit benefits \\
\hline
\end{tabular}

For Table 5: The Implicit Monetary Benefits of Increased Fitness Levels

\begin{tabular}{|l|c|c|}
\hline Implicit monetary benefits of reduced ACM probabilities & \\
\cline { 1 - 2 } (based on value of life inferred from market behavior) & & \\
\hline Assumed implicit value of life & & $\$ 3,000,000$ \\
\hline \multicolumn{2}{|c|}{ Assumed discount rate for implicit benefits } & $10.0 \%$ \\
\hline
\end{tabular}


The user also needs to estimate any purchased exercise inputs, effort costs, or other immediate costs or benefits of exercise for Table 3 on the costs of exercise inputs. Purchased exercise inputs would include health club fees and travel costs to exercise locations. Literature searches failed to turn up estimates of exercise effort costs, although studies on individuals' ratings of perceived exertion (RPE) exist [32, 33]. Those studies indicate that RPE increase sharply between moderate and high intensity exercise particularly from brisk walking to jogging and running. Thus, the input Table allows the user to assume different per hour effort costs for each intensity level of exercise. Again the user or advisor could ask how much money the individual would need just to expend different intensity levels of effort with no other costs, such as lost time or purchased exercise inputs, but also no health benefits from the effort. "Other yearly exercise costs or benefits" would include the risk of injury or any savings from a joint product exercise strategy which uses labor intensive technologies to perform daily tasks. Unpublished data indicate that walking or cycling to work instead of driving could generate capital operating and perhaps car ownership cost savings of up to approximately $\$ 5,000$ per year. Enter such savings as negative costs for each fitness level.

The discount rate at the bottom of this section of Table 1 poses a conceptual problem. If the individual wants to weigh the present values of the costs and benefits of a fixed commitment to a long-run exercise program, then they should discount the future exercise costs. Net present value analyses of business projects would discount future required capital investments. However, most exercise programs do not require future fixed capital commitments. Thus, to sustain the exercise program the individual must be willing to expend the high variable time and effort costs for each session year after year with no discounting. Therefore, this example compromises with a low 3 percent discount rate for the future exercise costs and does not increase it when comparing those exercise costs with future exercise benefits using higher discount rates for Table $\mathbf{5}$.

Next, the user data input table has an input section for Table 4 which analyzes the explicit monetary benefits of increased fitness. The user must enter estimates of expected yearly income during the follow-up period less any yearly insurance or survivor annuities if ACM occurs. This example uses an individual with an upper middle income of $\$ 80,000$ per year and good life insurance which would pay $\$ 50,000$ a year if ACM took place. Then the user must estimate the yearly HCC for a mid-fit individual after any insurance because the HCC index in Table $\mathbf{4}$ uses a mid-fit person as the base. This "after insurance" cost would include out-ofpocket expenditures for copayments and uncovered medical treatments which a typical mid-fit, middle aged individual in the user's insurance group or general medical cost situation would tend to incur. This example assumes $\$ 1,000$ a year for a mid-fit person in their 50's to early 60's who has good health insurance with low deductibles and copayments. A person without health insurance might assume $\$ 15,000$ a year, which would amount to $\$ 150,000$ over a ten year training and then a ten year follow-up period. This would cover possible major medical procedures, such as open heart surgery plus more routine yearly medical procedures.
The user must also assume a discount rate for the exercise induced reductions in the probability of lost income from ACM and HCC costs. The literature search yielded no consensus on a range of discount rates for long-run health benefits $[34,35]$. This example uses a low, real three percent because the explicit monetary value approach to exercise's long-run health benefits represents a financial approach. Three percent approximates the real, inflation adjusted longrun returns on diversified portfolios of investable assets, such as stocks, bonds, and diversified real estate [36].

For Table 5 the user or advisor must first choose an implicit value of life per year in the user data input Table $\mathbf{1}$. The implicit value of life approach uses market behaviors, or surveys, to estimate how people value their lives by requiring additional money for risky activities. The increased wages policemen tend to receive per year divided by the increased probability of mortality per year compared to similar but less risky work provides an example. These estimates range widely in the literature from around two to four million dollars per year for conservative estimates [30, 37], to up around seven million for calculating the overall social benefits of mitigating environmental and traffic hazards [35, $38,39]$. This example uses the midpoint of the conservative range on the assumption that people require less compensation for risks they have control over, such as exercise and fitness, and more compensation for risks they cannot control, such as environmental pollution [40]. Note that the literature does not support lowering the implicit value of life at older ages. For example, studies on the implicit value of life by age group and health [41] find only weak evidence that the implicit value of life drops with age. Moreover, people with chronic heart or lung disease or cancer seem willing to pay as much to reduce their risk of dying as people without these diseases.

Next, the user needs to choose a discount rate to calculate the present value of these cumulative reductions in the implicit cost of ACM. Again, no consensus on discount rates for long-run health exist [34, 35]. However, from a behavioral standpoint, it seems reasonable to assume that many people have some time-inconsistent preferences [42]. That is, at the present time they prefer not to exercise, but years later they wish they had exercised and stayed in better physical condition. This example arbitrarily uses a ten percent discount rate to reflect some time inconsistent preferences.

\section{The Model Output Tables}

The following explains how the model output tables use the data inputs to generate estimates of exercise benefits, costs, and net benefits. This section also suggests other changes more advanced users could make in the key parameters of the model. Again, note that throughout the model shaded cells indicate individualized data from the user data input (Table 1), and non-shaded cells contain calculations or assumptions about key parameters in the model.

\section{The Biological Production Functions Between Exercise, Fitness, and Long-Run Health}

Table 2 presents the production functions between longrun fitness, health, and exercise inputs in tabular form. See [2] for the graphs. The five fitness levels are listed across the top in metabolic equivalent units (METS) and the resulting 
Table 2. Production Functions Between Fitness, Long-Run Health, and Exercise Inputs

\begin{tabular}{|c|c|c|c|c|c|c|c|}
\hline & \multirow{2}{*}{\multicolumn{2}{|c|}{ Descriptions }} & \multicolumn{5}{|c|}{ Peak Fitness Levels (in METs*) } \\
\hline & & & Very Low & Low & Mid & High & Very High \\
\hline \multicolumn{8}{|c|}{ Production functions between fitness and long run health indicators } \\
\hline \multicolumn{2}{|c|}{ Probability of ACM during follow-up period by fitness level (males $55 \mathrm{y}+/-12$ ) } & & $40.0 \%$ & $25.0 \%$ & $17.5 \%$ & $12.5 \%$ & $8.5 \%$ \\
\hline \multicolumn{2}{|l|}{ Number of years in follow-up benefit period } & 10 & & & & & \\
\hline \multicolumn{2}{|l|}{ Less the average year in which ACM occurs, if it occurs } & 6 & & & & & \\
\hline \multicolumn{2}{|c|}{ Equals the average years lost from ACM during follow-up if ACM occurs } & 4 & & & & & \\
\hline \multicolumn{2}{|c|}{$\begin{array}{l}\text { Times probability of ACM equals expected years of life lost during } \\
\text { follow-up }\end{array}$} & & 1.6 & 1.0 & 0.7 & 0.5 & 0.3 \\
\hline \multicolumn{2}{|l|}{ Cumulative point drop in the HCC index from very low fit } & & 0.0 & 60.0 & 100.0 & 112.5 & 125.0 \\
\hline \multicolumn{8}{|c|}{$\begin{array}{l}\text { Threshold exercise intensities of } 30 \text { minute sessions to achieve different fitness } \\
\text { levels: }\end{array}$} \\
\hline \multicolumn{2}{|c|}{ Description of threshold exercise intensity to reach each fitness level } & & sedentary & light & moderate & vigorous & hard \\
\hline \multicolumn{2}{|c|}{ Approximate percent of age adjusted maximum heart rate (HR) (220 minus age) } & & $50 \%$ & $60 \%$ & $70 \%$ & $80 \%$ & $90 \%$ \\
\hline \multicolumn{8}{|l|}{$\begin{array}{l}\text { Time requirements for exercise inputs including travel and } \\
\text { clothes changing time }\end{array}$} \\
\hline \multicolumn{2}{|l|}{ Hours per session } & & 0 & 0.5 & 0.75 & 1 & 1.75 \\
\hline
\end{tabular}

* Metabolic equivalent units where one MET equals energy body expends while at rest or 3.5 milliliters of oxygen per kilogram of body weight

**Do not include continued training during the follow-up period. Such training would generate health benefits in another follow-up period.

probabilities for ACM [21] and a HCC index [3] are listed below. These are useful for intuitive long-run health planning with exercise and essential for estimating the costs and benefits of exercise for more quantitative analysis. The production functions indicate that increased fitness levels produce very important long-run health outputs for middle to early old-aged individuals. The probability of ACM drops from 40 percent over ten year follow-up periods for very low-fit to under 20 percent for mid-fit and under ten percent for very high-fit males. The HCC index also drops in half for increasing fitness from very low-fit to mid-fit and another 25 percent to very high-fit. These rates of drop in ACM and a HCC index apply to women [20] and to men with known heart disease [21], although the absolute values may vary. For example, middle-aged women would have lower absolute probabilities of ACM. More advanced users could program these changes into Table 2 .

The rows with the "Cumulative percentage point drop in probability of ACM...and point drop in the HCC index" provide the basis for estimating the long-run health benefits of individual exercise programs and require some careful explanation and consideration. The model attempts to estimate how an exercise program would increase an individual's fitness level above the level that individual would ex- pect to exhibit at the start of a follow-up period without the exercise program. The example in Table 2 assumes the individual would exhibit a very low-fit level at the follow-up period resulting from a sedentary life style. Therefore, any exercise program from light to very high intensity would increase fitness levels at the start of a follow-up period and reduce the probability of ACM and the HHC index during the follow-up. For example, a moderate intensity exercise program would have a 22.5 percentage point reduction in ACM and a 100 point reduction in the HCC index. However, if the individual would expect to arrive at the follow-up period in low or even mid-fit condition through daily activities and genetic disposition without any formal exercise program, the cumulative reductions in ACM and HCC would be substantially less. That is, for a person who expected to arrive at the start of the follow-up period in low-fit condition a moderate exercise program would only increase their fitness from low to mid-fit. This increase would only drop their probability of ACM by 7.5 percentage points and their HCC index by 40 points.

The very low-fit without an exercise program assumption remains reasonable for many individuals and uses of the model. Although 55-year-old men tend to exhibit mid-fit levels [21] in a society where surveys indicate less than 50 
percent of them engage in even consistent moderate exercise, by age 65 non-exercising males tend to drop to the low and very low-fit levels (see figure 3 in [2]). Nevertheless, for a rigorous application of the model users who would expect to arrive at the follow-up period in higher-fitness levels through their normal activities without an exercise program would have to put a zero under the fitness level they expected in the "Cumulative percentage point drop in ACM" row. The model would automatically make the change in the "Cumulative point drop in the HCC index" row. Then only exercise programs with intensities high enough to achieve even higher levels of fitness at follow-up would generate further reductions in ACM probabilities and the HCC index. To return to the original assumption of very low-fit at the start of the follow-up period, the user must copy the formulas in the higher fit "cumulative percentage point drop in ACM" cells back to the low and mid-fit columns.

The next lines of Table 2 estimate the years of life at risk to ACM for each fitness level and how exercise, which increases fitness, lowers that risk. Note that these are years of life at risk during the follow-up period only and do not relate directly to years of life expectancy. First, the model assumes the number of years in the follow-up period and the average year during that follow-up period when ACM takes place if it occurs based on the Myers study [21], which is similar to other studies in the area $[18,19,22]$. Advanced users, however, could change those assumptions. The difference between the two provides an estimate for the number of years which would be lost on average if ACM took place during the follow-up period. Thus, the average years lost during the follow-up period, if ACM takes place, equal four. The next line multiplies those four years times the probability of ACM for each fitness level to calculate expected years of life lost for each fitness level.

"Expected" involves a statistical concept concerning the number of deaths during the follow-up period over a number of trials or individuals given the probability of ACM. For example, in a group of 100 very low-fit individuals the model expects 40 to die during the follow-up period, and on the average those who die lose four years of life during the ten year follow-up period. Thus, the model would expect the group to lose 160 years of life (40 individuals $\mathrm{x} 4$ years) and would expect one individual to lose 1.6 years of life (160 years/100 individuals) during the follow-up.

The cumulative drop in those expected years of lost life in the next line provides a measure of the benefits of increasing fitness levels which the following tables will use. Again, note that these reductions in expected years of lost life do not indicate increased years of expected life since they only cover one follow-up period. The increase in life expectancy from increased exercise and fitness levels would be considerably higher for a middle-aged individual who trained up through at least early old age (65-75) because he or she would reduce the risk of ACM over more than one ten year follow-up period.

Next, the HCC index in Table 2 provides a framework for estimating the reduction in $\mathrm{HCC}$ as fitness levels increase. The index comes from one large $(\mathrm{n}=881)$ study [3] which related fitness levels to HCC for a group of mainly men with an average age of 59 years (SD +/-12) who obtained their general health care at a Veterans Administration facility. Most of the subjects had cardiovascular disease (CVD) risk factors, but few had overt evidence of CVD. The study conducted initial stress tests to establish fitness levels and then collected HCC data for one year. Table 2 converts the study's HCC for the five different fitness levels into a HCC index with the base at mid-fit equaling 100 . Such an index allows individuals with different insurance and health care needs to estimate how exercise could reduce their HCC. This index exhibits a similar rate of decline with increased fitness levels as the ACM production function. The cumulative drop in the HCC index on the next line provides another indicator of the long-run health benefits of exercise which increases and/or maintains age limited fitness levels.

The next section of Table 2 presents descriptions of threshold exercise intensities for approximately 30 minute exercise sessions three to six times per week required to increase and/or maintain each fitness level. The first line puts a name on the intensity level, such as "moderate" or "vigorous". The next line quantifies that level in terms of heart rates as approximate percent of age adjusted maxima which individuals can estimate with the formula, 220 - age. Note, as indicated above, substantial evidence indicates that individuals can use interval training to achieve these threshold levels. They do not need to exercise continuously for 30 minutes at the threshold level.

The last section of Table 2 calculates the time costs of exercise for different exercise intensities and resulting fitness levels. This section takes the user input data for hours per session and sessions per week to calculate the total exercise hours per year and over the training period. The last two lines of Table 2 illustrate how these typical exercise time costs per session amount to over four to five work weeks per year and approximately a year of work over a ten year training period. Thus, if a person had a moderate to high value of time per hour, the monetary costs of even moderate exercise can become very high.

\section{The Monetary Costs of the Exercise Inputs for Long-Run Health}

Table 3 provides a framework for estimating the monetary costs of the exercise inputs. These are useful for cost effective analyses of alternative long-run health inputs and for comparing exercise costs to the monetary benefits for benefit cost analysis. The bottom lines of Table 3 suggest that exercise remains a relatively high cost long-run health input. For this user's data assumptions, costs fall in the $\$ 40,000$ to $\$ 60,000$ dollar range over a ten year training period, or in the $\$ 4,000$ to $\$ 6,000$ per year range.

To arrive at this estimate the framework takes the users' assumed dollar value per hour for time spent exercising and other exercise costs less any savings on capital intensive technologies, such as riding a bike instead of driving a car for transportation, from the user data input Table 1. Table $\mathbf{3}$ then sums up the total yearly exercise time and other costs and takes their present values to the beginning of the followup period using the embedded present value formula in the spreadsheet. This example only includes the time costs of exercise with no purchased inputs, effort costs, or other yearly exercise costs or benefits. Note that the table does not count any exercise into the follow-up period as a cost. The exercise in the follow-up period would generate benefits in 
Table 3. The Monetary Costs of Exercise Inputs Needed to Increase Fitness Levels and Long-Run Health

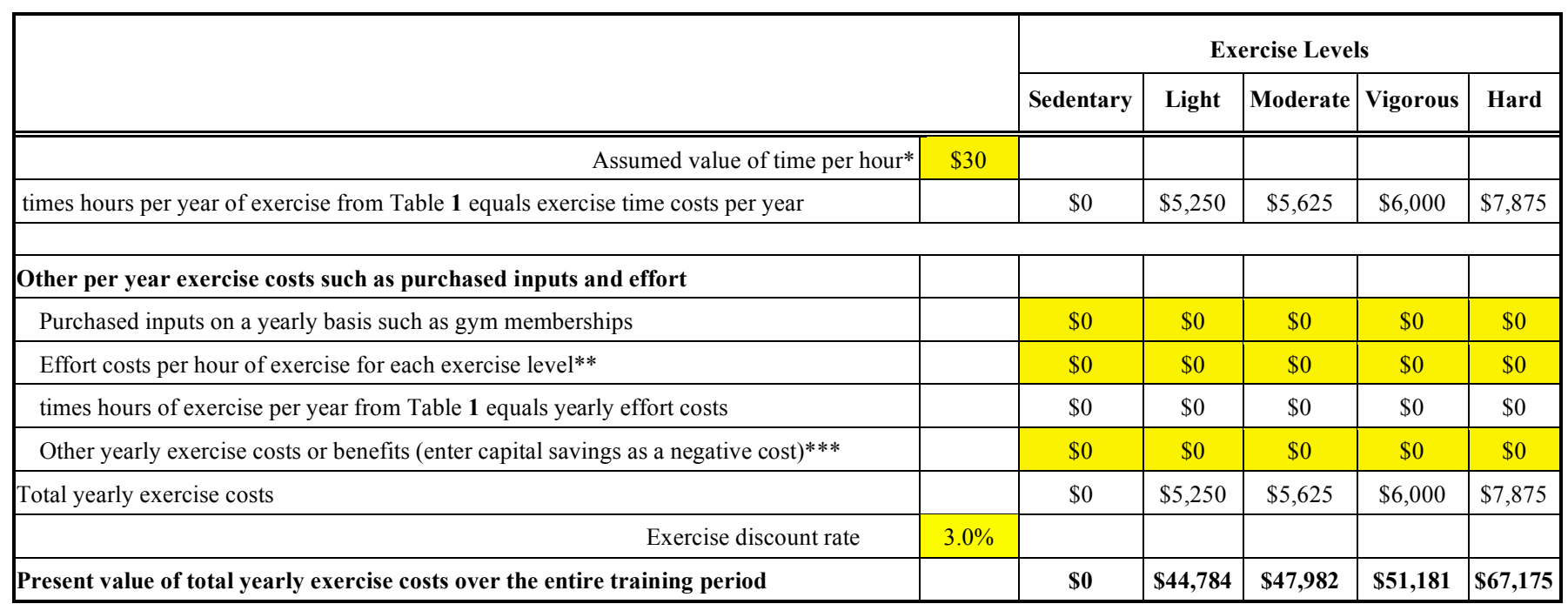

*Could range from zero through average wage rate to high marginal value (e.g., what individual would need to stop present activity for an hour).

**Dollars per hour would need just for the exercise effort if no health benefits expected.

$* * *$ These include operating and capital ownership savings when a person engages in a joint product exercise strategy using labor intensive technology such as walking or bicycling to work instead of driving and perhaps owning another household car.

another follow-up period not included in this analysis. Then Table 3 brings down the low 3 percent discount rate from the user data input Table $\mathbf{1}$.

\section{The Explicit Monetary Long-Run Health Benefits of Exercise and Increased Fitness}

Table 4 provides a framework for estimating the explicit monetary benefits to exercise, increased fitness, and increased long-run health. These include reductions in the risk of losing household income through ACM or imposing HCC on the household. This represents a financial approach to the value of exercise and expected increased long-run health. This financial approach can help analyze exercise versus insurance decisions and the joint product approach to exercise using labor intensive technology with capital savings, such as walking or cycling for short distance transportation rather than driving. The data for this example individual with good insurance yields negative net benefits in the bottom line because of high exercise time costs. A person without health and life insurance or with a joint product exercise strategy could generate substantial positive net benefits to an exercise program as Table 6 indicates.

To calculate the net benefits, the table first takes the user's expected income and any expected yearly life insurance or survivor's annuity to the household from the user data input (Table 1). The difference equals lost income per year during the follow-up period if ACM occurs. Then Table 4 multiplies that yearly net loss times the "expected years of life lost" during the follow-up period for each fitness level from Table 2 to generate expected lost income during the follow-up period. The "cumulative reduction in lost income" line subtracts the expected lost income for each higher fitness level from the expected loss for persons in the very lowfit category in this example. This represents an estimated lump sum future benefit to the exercise which increases and/or maintains the individual's fitness to each level above the expected base. Again, note that the user might arrive at the start of the follow-up period with a low to even moderate fitness level without an exercise program. A rigorous analysis of that assumption would require adjustments to the model as described above.

Table 4 then takes the user's assumed discount rate from the data input (Table 1) to discount those cumulative lump sum reductions in expected lost income from ACM back to the start of the training period using the formula:

\section{the cumulative lump sum reduction in the prob- ability in expected lost income divided by $(1+$ the discount rate) raised to the number of years in the training period plus the average number of years into the follow-up period when $\mathrm{ACM}$ occurs if it occurs.}

The example in Table 1 uses ten years for the training period and six years before ACM occurred on the average in the follow-up period. This yields a 16 year cycle over which Table 4 discounts the monetary benefits of reduced ACM for each fitness level.

The middle section of Table $\mathbf{4}$ uses a similar process to estimate how exercise and increased fitness can lower longrun HCC. The table takes the user's estimate of the yearly HCC for an individual in the mid-fit group from Table 1. Then Table 4 multiplies that yearly HCC for a mid-fit individual by the HCC index in Table 2 and divides by 100 to generate the expected HCC per year for each fitness level. This example continues to bring down the very low-fit level without exercise assumption. Thus, the cumulative benefits range from $\$ 600$ to $\$ 1,250$ per year in expected reduced HCC for exercise programs, which raise and/or maintain fitness levels from that very low level.

To calculate the present value of this yearly reduction in HCC the table uses a more complex set of formulas than for the reduction in ACM, which was a lump sum. First, the table uses the embedded present value formula in the spreadsheet to discount the stream of yearly HCC benefits back to the start of the follow-up period. This formula uses the full follow-up period because individuals in their $50 \mathrm{~s}$ and $60 \mathrm{~s}$ 
Table 4. The Explicit Monetary Benefits of Increased Fitness Levels During the Follow-Up Period

\begin{tabular}{|c|c|c|c|c|c|c|}
\hline & & Very Low & Low & Mid & High & Very High \\
\hline Expected yearly income during the follow-up period & $\$ 80,000$ & & & & & \\
\hline less yearly life insurance payout or survivor annuity if ACM occurs & $\$ 50,000$ & & & & & \\
\hline \multicolumn{2}{|l|}{ times expected years lost in Table 2 , if ACM occurs, equals expected lost income* } & $\$ 48,000$ & $\$ 30,000$ & $\$ 21,000$ & $\$ 15,000$ & $\$ 10,200$ \\
\hline Cumulative reduction in lost income as fitness increases from** & very low-fit & $\$ 0$ & $\$ 18,000$ & $\$ 27,000$ & $\$ 33,000$ & $\$ 37,800$ \\
\hline Present value at start of training period with discount rate of & $3.0 \%$ & $\$ 0$ & $\$ 11,217$ & $\$ 16,826$ & $\$ 20,565$ & $\$ 23,556$ \\
\hline \multicolumn{2}{|l|}{ times HCC index in Table 2 divided by 100 equals expected HCC per year } & $\$ 2,000$ & $\$ 1,400$ & $\$ 1,000$ & $\$ 875$ & $\$ 750$ \\
\hline Cumulative reduction in yearly $\mathrm{HCC}$ as fitness increases from** & very low-fit & $\$ 0$ & $\$ 600$ & $\$ 1,000$ & $\$ 1,125$ & $\$ 1,250$ \\
\hline \multicolumn{3}{|l|}{ Present value at start of training period, same discount rate $* * * *$} & $\$ 2,834$ & $\$ 4,723$ & $\$ 5,313$ & $\$ 5,904$ \\
\hline \multicolumn{2}{|l|}{ Present value of explicit $\mathrm{ACM}$ and $\mathrm{HCC}$ benefits at start of training period } & $\$ 0$ & $\$ 14,051$ & $\$ 21,548$ & $\$ 25,878$ & $\$ 29,459$ \\
\hline \multicolumn{2}{|l|}{ Less present value of exercise input costs where a negative cost is a benefit**** } & $\$ 0$ & $\$ 44,784$ & $\$ 47,982$ & $\$ 51,181$ & $\$ 67,175$ \\
\hline \multicolumn{2}{|c|}{ Net explicit long-run health benefits } & $\$ 0$ & $-\$ 30,733$ & $-\$ 26,434$ & $-\$ 25,303$ & $-\$ 37,716$ \\
\hline
\end{tabular}

generally would expect to survive the full follow-up period and incur some HCC, although a risk of ACM exists. Then the spreadsheet discounts that present value at the start of the follow-up period back to the start of the training period using the formula:

Present value of reduced HCC at the start of the follow-up period divided by $(1+$ discount rate $)$ raised to the number of years in the training period.

The last section of Table 4 sums the present values of the explicit monetary benefits of reductions in ACM and HCC for each fitness level. Next Table 4 subtracts the present value of the exercise costs found in Table 2 to generate an estimate of net explicit long-run health benefits. Again, note that joint product exercise strategies, such as bicycling to work instead of driving, may produce savings in capital ownership and operating costs which could increase the overall net benefits of exercise substantially as Table 6 indicates.

\section{The Implicit Monetary Long-Run Health Benefits of Ex- ercise and Increased Fitness}

Table 5 provides a framework for estimating the implicit monetary benefits of reductions in the risk of ACM from exercise and increased fitness. This broader approach may be useful for insights to exercise behavior, or lack thereof, and optimal individual exercise strategies, as well as over-all social benefit cost analysis. The implicit or statistical value of life approach rests on market behavior and questionnaires about hypothetical behavior, such as the higher salaries police personnel receive or would need, other things equal, to compensate for the higher risk of death and injury. This involves a much broader approach which attempts to include the overall utility or satisfaction with life and disutility from risking and perhaps losing it. This example yields net benefits in the $\$ 300$ to $\$ 700$ thousand dollar range, but other assumptions can lead to much lower estimates (see summary Table 6). 
Table 5. The Implicit Monetary Benefits of Increased Fitness Levels During the Follow-Up Period

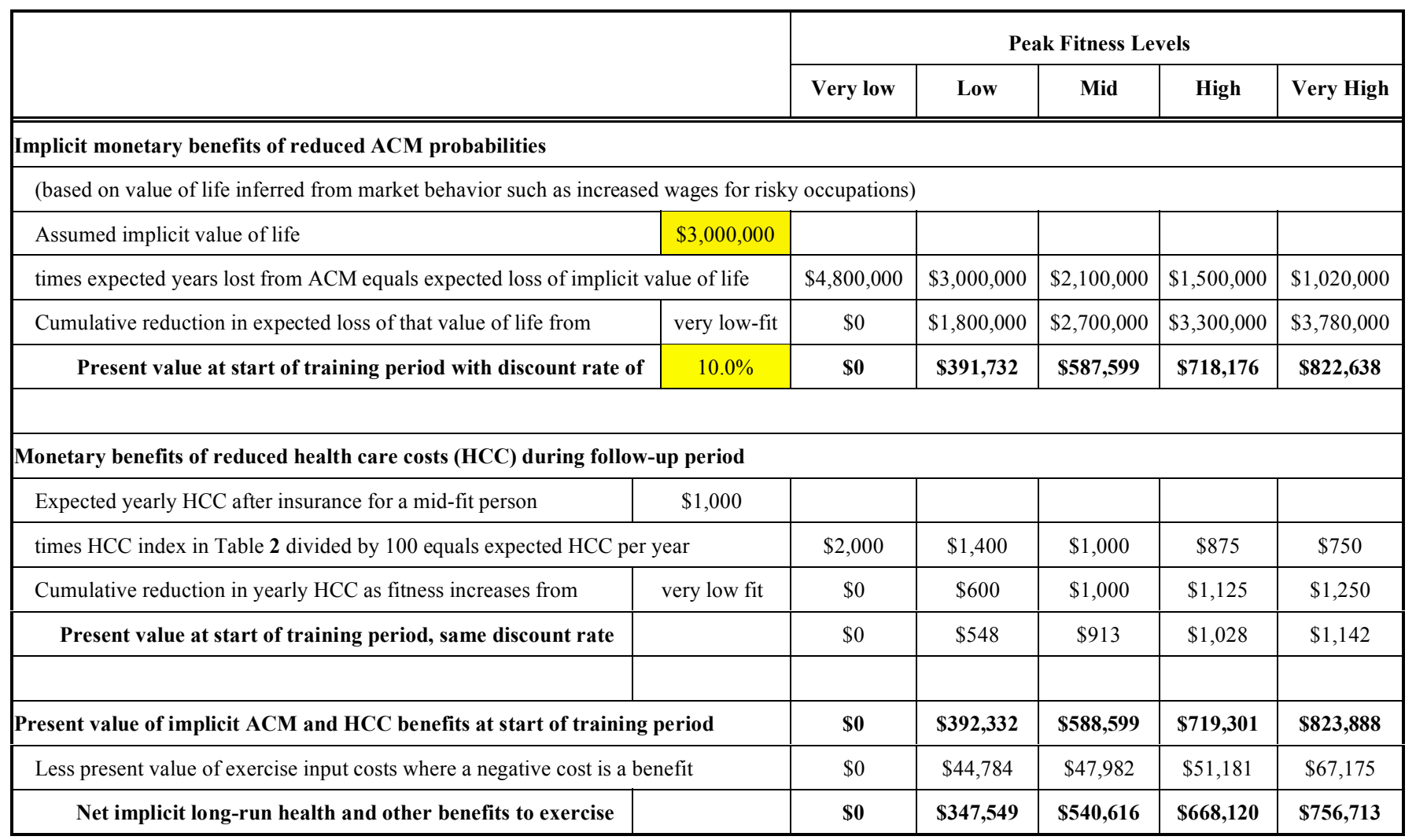

See notes under Table 3 .

To arrive at these net benefits Table 5 brings down the user's assumed implicit value of life per year from the user data input Table 1. Then Table 5 multiplies that value times the number of years of life the individual would expect to lose from ACM at each level of fitness during the follow-up period from Table 1. This yields an expected implicit monetary value for loss of life to ACM for each fitness level. The next line calculates the cumulative reduction in these possible losses from the base line fitness the user assumes the individual would exhibit at the start of the follow-up period without an exercise program. Next the table takes the user's discount rate for the reduction in the implicit value of reduced ACM to calculate the present value of that reduction.

The following rows in Table $\mathbf{5}$ just repeat the explicit values of the reductions in HCC from Table 4. Note that their present values fall below those in Table 4 because the discount rate in Table 4 equals three percent for the financial approach. Table 5, however, uses ten percent for the implicit approach with time inconsistent preferences. Including reductions in HCC may create some double counting since higher compensation for riskier jobs must cover some implicit valuation of increased accident, injury, or illness expectations. On the other hand, HCC associated with riskier employment may be infrequent and different from routine HCC estimated in Table 1. Thus, the two may not overlap much. Moreover, reductions in the implicit value of possible lost life dwarf the reductions in HCC, particularly for persons with good health insurance.

Finally, Table 5 sums the present values of the reduction in ACM losses and in HCC, subtracts the exercise costs, and generates a net long-run health benefit. With the above as- sumptions these net benefits become very large in the $\$ 300$ to $\$ 700$ thousand dollar range. Model users probably should not interpret such results literally because they conflict so strongly with the observed lack of exercise behavior, and other assumptions could reduce the net benefits to near zero. For example, higher discount rates for time-inconsistent preferences plus inclusion of effort costs can reduce net implicit benefits to modest or negative levels (see Table 6). Rather, model users should focus on how net benefits change as time and other exercise costs, assumptions about time inconsistent preferences, or exercise strategies change.

\section{Summarizing the Various Assumptions and Scenarios}

Table 6 allows the user to summarize the net benefits to exercise using different assumptions such as those discussed above. The user can employ the spreadsheet "copy" and "paste special" commands and then select "values" to quickly capture the results of each scenario. Note that the user needs to take care to record all the changed variables in all the tables for each scenario. For example, if one scenario changed the per hour time requirements or effort costs, the user should either return them to their old values or note them in a new scenario, which also changes other variables such as the assumed value of life or yearly HCC.

Table 6 shows that the explicit value of life approach generated negative net explicit benefits to exercise in Table 4 for a person who would become very low-fit at the follow-up period without an exercise program, but who had good health insurance. However, that explicit value of life approach can generate positive net benefits if that same individual does not have life and health insurance. The same 
Table 6. Summary of Net Exercise Benefits for Each Fitness Level with Different Data Assumptions

\begin{tabular}{|c|c|c|c|c|c|}
\hline & \multicolumn{5}{|c|}{ Peak Fitness Levels } \\
\hline & Very Low & Low & Mid & High & Very High \\
\hline \multicolumn{6}{|l|}{ Explicit value of life or financial approach } \\
\hline Example in Table 4, very low-fit, good insurance, $3 \%$ discount rate & $\$ 0$ & $-\$ 30,733$ & $-\$ 26,434$ & $-\$ 25,303$ & $-\$ 37,716$ \\
\hline Same but no life or health insurance, $\$ 15,000$ per year HCC & $\$ 0$ & $\$ 27,635$ & $\$ 67,730$ & $\$ 83,358$ & $\$ 84,196$ \\
\hline $\begin{array}{l}\text { Same but would be low-fit, not very low-fit, at follow-up without exercise pro- } \\
\text { gram }\end{array}$ & $\$ 0$ & $-\$ 44,784$ & $-\$ 4,689$ & $\$ 10,939$ & $\$ 11,777$ \\
\hline $\begin{array}{l}\text { Same but saves } \$ 5,000 \text { a year in capital costs with a joint product exercise strat- } \\
\text { egy }\end{array}$ & $\$ 0$ & $-\$ 2,113$ & $\$ 37,962$ & $\$ 53,590$ & $\$ 54,428$ \\
\hline \multicolumn{6}{|l|}{ Implicit value of life or utility approach } \\
\hline $\begin{array}{l}\text { Example in Table 5, implicit value of life } \$ 3,000,000 \text {, very low-fit, } 10 \% \text { discount } \\
\text { rate }\end{array}$ & $\$ 0$ & $\$ 347,549$ & $\$ 540,616$ & $\$ 668,120$ & $\$ 756,713$ \\
\hline Same with $25 \%$ discount rate & $\$ 0$ & $\$ 6,482$ & $\$ 29,016$ & $\$ 42,831$ & $\$ 40,472$ \\
\hline Same with effort cost of $\$ 5, \$ 10, \$ 30$, and $\$ 40$ per hour as intensity increases & $\$ 0$ & $-\$ 1,025$ & $\$ 12,936$ & $-\$ 8,607$ & $-\$ 49,436$ \\
\hline
\end{tabular}

assumptions, but with the person potentially arriving at age 55 in low instead of very low-fit condition, would reduce the net benefits to an exercise program to negative or modest levels again (scenario 3 under the "Explicit value of life approach" in Table 6). However, a joint product approach to exercise with savings on capital could raise net benefits back to substantial levels.

The implicit value of life approach in Table 6 first shows the very high net benefits that approach generated in Table $\mathbf{5}$ for a person who would have become very low-fit at the follow-up period without exercise. Those net benefits can drop to low levels if the individual has high time inconsistent preferences and a 25 percent discount rate. If the person also has high exercise effort costs, the net benefits become negative for most fitness levels (last line in Table 6). Lack of knowledge about the exercise's biological benefits, although not shown, could also lower the expected net benefits. Again users should focus on the changes in net benefits with different assumptions and exercise strategies rather than their absolute levels given the wide ranges for the implicit value of life.

\section{DISCUSSION}

The construction of an individual benefit-cost framework of exercise based on computer spreadsheets seems feasible. Expanding the framework into a model based on more solid and easier-to-understand biological production functions, which use objective measures of fitness and absolute probabilities rather than reported physical activity and relative risk, also seems feasible. A user input table feeds to output tables with clear bottom line indicators of exercise costs and benefits. The tables in this paper provide a model for various purposes from health planning, through financial analyses of exercise based on explicit economic benefits, to exercise behavior analysis based on implicit value of life estimates. The biological studies underlying the ACM and HCC production functions have become very solid. The monetary estimates of the exercise costs, benefits, and net benefits remain less certain. Nevertheless, the model may provide useful insights into the costs and benefits of exercise, the barriers to exercise, and optimal individual exercise strategies.

For example, the model clearly illustrates the paradox that exercise constitutes a very powerful health input, yet relatively few people use that input particularly at the higher intensity levels. Table $\mathbf{2}$ indicates that the probability of ACM drops from approximately 40 to 20 percent over ten years for persons in the 50 and 60 year-old age groups who exercise enough to keep their fitness levels at mid-fit levels compared to those who drop to very low-fit levels. Table $\mathbf{5}$ indicates that people put a very high implicit value on their lives even into old age. Thus, those drops in ACM can generate very high monetary benefits. Yet surveys of exercise among U.S. adult populations indicate that less than half engage in regular moderate exercise to generate mid-fit levels and that portion drops with age $[43,44]$. Less than ten to twenty percent engage in vigorous to hard intensity aerobic exercise to produce high and very high-fit levels which can drop the probability of ACM to under ten percent.

The model also suggests or reinforces several possible explanations for this paradox and strategies to increase exercise on an individual and society level. First, the model suggests that individuals' lack of knowledge about the high probable biological benefits of exercise may prevent them from perceiving the high potential implicit monetary benefits to exercise. Most individuals may view exercise as just one of many recommended short-run and long-run health inputs. Although the literature indicates that most people understand the public health exercise recommendations [45], which have been widely publicized [46, 47], no evidence exists they understand how exercise which increases fitness drops the probability of ACM and HCC dramatically as Table 2 indicates. The public health exercise recommendations have emphasized decreases in relative risk rather than the more easily understood and relevant drops in the absolute probability of ACM. 
Second, the model indicates that the present high time and perhaps effort costs of exercise probably swamp the present value of even very high long-run future benefits particularly with time inconsistent preferences and high discount rates. Table 3 shows that just the present time costs of regular exercise can reach high levels in the four to six thousand dollar per year range without counting the effort and other possible costs. Thus, individuals may exhibit rational, satisfaction maximizing behavior by not using the exercise input for long-run health. Relying on medications and surgical interventions probably constitutes the low cost approach particularly for persons with good health insurance. To make the exercise input a rational choice, the model suggests that individuals and society must find ways of lowering the immediate costs of exercise. This reinforces the active living [48], or joint product [12], strategies. Combining exercise with transportation by walking or cycling for short trips can reduce the time costs of exercise and perhaps capital costs of owning an extra household car [49]. Similar savings can result from many other activities, such as kayaking instead of power boating, mowing small to mid-sized lawns with hand pushed reel mowers, leaf raking, gardening with hand tools, and cross country versus downhill skiing. Unpublished analyses of such joint product strategies using this individual exercise model often yield high net benefits. For example, such strategies can increase the explicit net benefits by approximately $\$ 50,000$ from the negative $\$ 20,000$ range to the positive $\$ 30,000$ range for persons with good insurance. The joint product strategy also can increase the implicit value of life net benefits by approximately $\$ 40,000$. The yearly capital savings can amount to $\$ 5,000$ a year or more, which can provide more immediate reinforce for exercise by increasing current consumption in other areas or by providing current savings for retirement. For example, over 20 years $\$ 5,000$ a year saved at a 3 percent real interest rate generates over $\$ 130,000$ and over 40 years over $\$ 370,000$ in current purchasing power.

Finally, the model reinforces how societal level or environmental programs such as bike and walkways, restrictions on driving, and information campaigns can encourage these joint product approaches to exercise. Such programs can lower the perceived accident risk and perhaps time costs of walking and cycling for transportation as they increase the relative time and monetary costs of driving. Noise and air pollution restrictions can make reel and electric push lawn mowers more attractive than gas powered, self propelled mowers. Restrictions on boat size and speeds on smaller waterways not only can provide ecological protection, but also can make paddling and rowing more attractive.

While this benefit cost model of exercise does provide or reinforce important insights to the lack of exercise and strategies for overcoming them, the model has a number of limitations. First, the model only analyzes individual exercise behaviors and programs. To extend the model to the benefits and costs of societal level exercise programs and facilities would require several modifications. For example, users would need to predict how many individuals the exercise program or facility would induce to exercise and their age and gender demographics. Model users could not assume a proposed facility like a bikeway would cut ACM and HCC of projected facility users in half. Some facility users would have been exercising anyway and younger users would not realize the long-run health benefits for several decades. These considerations would tend to reduce the social benefits to exercise facilities. However, use of the implicit value of life approach could increase the social benefits over existing social benefit cost approaches based only on explicit benefits, such as reductions in lost income from premature mortality. The implicit long-run health benefits could be very high if the framework could use lower discount rates for facilities which provided other immediate benefits to exercise, such as getting to work or engaging in recreation. Such immediate benefits could over ride time inconsistent preferences for long-run health, and thus, justify lower discount rates.

A second limitation of the model involves the assumption of a ten year training period for an early middle-aged individual and then ten year health benefit follow-up period. Users can lengthen the training period for a younger person. This increases costs and lowers discounted future benefits. Thus, a younger person would tend to need more immediate benefits, such as the joint products approach, to find optimal exercise strategies. At the same time, the framework does not include more immediate health benefits of exercise and fitness which can increase net benefits for younger users. Prevention and control of adult onset diabetes provides an example. Nevertheless, the model suggests that exercise does not represent a good substitute for health and life insurance for younger individuals. The net explicit or financial benefits to exercise for a person without health and life insurance could be high in the $\$ 60,000$ to $\$ 80,000$ range. Still, substantial probabilities, at least approximating ten percent by the mid 50s, of ACM or major health care expenditures would remain which exercise could not eliminate. Insurance could cover those risks and give a much higher protection of household income and assets.

A third limitation of the model involves the assumption that an individual would arrive at the follow-up period in middle to old age exhibiting very low-fit condition without an exercise program. As indicated in the demonstration section many individuals may arrive at age 55 and a few at age 65 in mid-fit condition through daily physical activity without an exercise program. Thus, advanced users of the model may want to assess their current fitness levels and attempt to estimate how different proposed exercise programs would affect those fitness levels at the start of the follow-up period. Sedentary individuals could employ formal stress tests under medical supervision which committees of cardiologists have recommended for older and lower-fit persons [50]. Regularly exercising and more fit individuals can informally use MET computations on gym equipment to roughly estimate their fitness levels and changes in them. One approach involves starting to exercise at a comfortable MET level for two minutes and then raising that level and holding it for another two minutes. The highest two minute level approximates the individual's peak fitness in METS. Formal stress tests often express fitness levels in terms of oxygen up-take $\left(\mathrm{VO}_{2}\right)$. Individuals can convert those test results to METs by dividing measured or estimated $\mathrm{VO}_{2}$ by 3.5 since one MET equals 3.5 milliliters of $\mathrm{VO}_{2}$ per kilogram of body weight per minute.

Once individuals have estimates of their fitness level they can use figure 3 in [2] to roughly determine how this level would tend to drop with age given their general levels of 
physical activities. That figure suggests that by approximately age 50 individuals who quit obtaining exercise drop to very low-fit levels within five to ten years, even if they were in the very high-fit category. Those who continue exercising at moderate to light intensities fall into the mid-fit category. However, by age 65 to 70 they also fall into the low-fit and perhaps very low-fit categories particularly if they only engage in very light exercise. The literature behind figure 3 suggests that some master athletes can retard the drop in fitness by maintaining vigorous to hard exercise programs. These individuals may arrive at follow-up periods in their 60 s and even early 70 s in the high to very high-fit levels. The myocardial infarct (heart attack risk) for at least moderate to vigorous exercise intensity seems to remain low if the individual consistently exercises at those levels three to five days a week [51].

A fourth limitation with the model involves the lack of other long run health benefits to exercise and fitness besides reductions in ACM and HCC. For example, increased fitness and physical and mental functionality may allow one to work and earn longer and reduce the probability of expensive assisted living and even nursing home care. Numerous studies indicate a relationship between exercise, fitness, and other health benefits, such as reductions in CVD [52] and the control of diabetes. But these generally do not fit into a clear relationship with the five fitness levels listed in Table $\mathbf{2}$ or can not be estimated monetarily as easily as ACM or HCC. Studies on exercise, fitness, and mental functionality exist and could be very useful, but the relationships do not seem very large in the current studies $[53,54]$ and remain difficult to estimate in monetary terms. For an extensive summary of the documented health benefits of exercise see [55].

A final set of limitations with the model include the lack of more precise monetary estimates of exercise costs and health benefits for some key variables. These include the value of exercise time for different individuals at different time periods, the implicit value of life, and the discount rate to reflect time inconsistent preferences. As indicated under the demonstration of the biological production functions, questionnaires might help estimate a specific user's value of time and effort. Those questionnaires ideally should employ a conjoint approach where the individual must make choices between different alternative activities or amounts of money for different exercise time and effort expenditures [56, 57]. Questionnaires probably would not provide more reliable estimates for the implicit value of life approach for specific individuals, however. Thus, model users should not relay on the absolute values for the implicit value of life approach. Rather, they should use the approach to analyze how different exercise strategies could change the net benefits.

\section{CONCLUSION}

A widely accessible spreadsheet framework and model for estimating the individual monetary benefits and costs of exercise based on solid biological production functions between five levels of fitness and reductions in ACM and HCC seems feasible. These indicators of long run health capture many of the important health benefits of exercise at least for middle and early old age individuals and lend themselves to monetary estimates. Similar relationships on mental and physical functionality and diseases of younger adults, such as type 2 diabetes, would improve the model. The model also needs better data on some of the key monetary variables, such as the implicit value of life, effort costs, and discount rates to reflect time inconsistent preferences. The assumption of ten years of training and then a ten year follow-up period also may create some confusion.

Nevertheless the framework and model in this paper seem useful for a variety of purposes at the individual and perhaps small group level. These purposes include long-run health planning (Table 2), cost effective analysis of exercise (Table 3), financial estimates of exercise benefits including the joint product exercise strategy (Table 4), and utility analysis for exercise behavior using implicit value of life estimates (Table 5). Preliminary work on joint product approaches indicates that they can generate very high net benefits even in the explicit value of life or financial approach. Society level projects like walk and bikeways can lower the costs of exercise, increase the net benefits to exercise, and theoretically increase participation in consistent daily exercise. This individual framework and model of the individual benefits and costs of exercise may provide a more solid foundation for social benefit cost analyses of such projects. However, this would require additional data on how much increased exercise the facility would generate and the ages of the expected users.

\section{ACKNOWLEDGEMENTS}

The authors wish to thank Dr. William J. Trainor Jr., Department of Economics and Finance at East Tennessee State University for extensive and valuable comments on the manuscript.

\section{REFERENCES}

[1] Franklin BA. Fitness: the ultimate marker for risk stratification and health outcomes? Prev Cardiol 2007; 10: 42-5.

[2] Everett MD, Kinser A, Ramsey MW. Training for old age: production functions for the aerobic exercise inputs. Med Sci Sports Exerc 2007; 39: 2226-33.

[3] Weiss JP, Froelicher VF, Myers JN, Heidenreich PA. Health-care costs and exercise capacity. Chest 2004; 126: 608-13.

[4] Hirshleifer J. The expanding domain of economics. Am Econ Rev 1985; 75: 53-68.

[5] Colditz GA. Economic costs of obesity and inactivity. Med Sci Sports Exerc 1999; 31: 663-7.

[6] Cooper K, Brailsford SC, Davies R, Raftery J. A review of health care models for coronary heart disease interventions. Health Care Manag Sci 2006; 9: 311-24.

[7] Hatziandreu EI, Koplan JP, Weinstein MC, Caspersen CJ, Warner KE. A cost-effectiveness analysis of exercise as a health promotion activity. Am J Public Health 1988; 78: 1417-21.

[8] Jones TF, Eaton CB. Cost-benefit analysis of walking to prevent coronary heart disease. Arch Fam Med 1994; 3: 703-10.

[9] Sevick MA, Dunn AL, Morrow MS, Marcus BH, Chen GJ, Blair SN. Cost-effectiveness of lifestyle and structured exercise interventions in sedentary adults: results of project ACTIVE. Am J Prev Med 2000; 19: 1-8.

[10] Shephard RJ. A critical analysis of work-site fitness programs and their postulated economic benefits. Med Sci Sports Exerc 1992; 24: 354-70.

[11] Wang G, Macera CA, Scudder-Soucie B, Schmid T, Pratt M, Buchner D. A cost-benefit analysis of physical activity using bike/pedestrian trails. Health Promot Pract 2005; 62: 174-9.

[12] Everett MD. Raising exercise levels among adult Americans. J Behav Econ 1980; 9: 55-72.

[13] Pratt M, Macera CA, Sallis JF, O'Donnell M, Frank LD. Economic interventions to promote physical activity: application of the SLOTH model. Am J Prev Med 2004; 27: 136-45. 
[14] Sturm R. The economics of physical activity: societal trends and rationales for interventions. Am J Prev Med 2004; 27: 126-35.

[15] Sturm R. Economics and physical activity: a research agenda. Am J Prev Med 2005; 28: 141-9.

[16] King AC. Community and public health approaches to the promotion of physical activity. Med Sci Sports Exerc 1994; 26: 1405-12.

[17] Noar SM. A health educator's guide to theories of health behavior. Int Q Commun Health Educ 2005; 24: 75-92.

[18] Blair SN, Kohl HW,3rd, Paffenbarger RS, Jr, Clark DG, Cooper KH, Gibbons LW. Physical fitness and all-cause mortality: a prospective study of healthy men and women. JAMA 1989; 262: 2395-401.

[19] Laukkanen JA, Lakka TA, Rauramaa R, et al. Cardiovascular fitness as a predictor of mortality in men. Arch Intern Med 2001; 161: 825-31.

[20] Mora S, Redberg RF, Cui Y, et al. Ability of exercise testing to predict cardiovascular and all-cause death in asymptomatic women: a 20-year follow-up of the lipid research clinics prevalence study. JAMA 2003; 290: 1600-7.

[21] Myers J, Prakash M, Froelicher V, Do D, Partington S, Atwood JE. Exercise capacity and mortality among men referred for exercise testing. N Engl J Med 2002; 346: 793-801.

[22] Sui X, LaMonte MJ, Laditka JN, et al. Cardiorespiratory fitness and adiposity as mortality predictors in older adults. JAMA 2007; 298: 2507.

[23] DeBusk RF, Stenestrand U, Sheehan M, Haskell WL. Training effects of long versus short bouts of exercise in healthy subjects. Am J Cardiol 1990; 65: 1010-3.

[24] Murphy MH, Hardman AE. Training effects of short and long bouts of brisk walking in sedentary women. Med Sci Sports Exerc 1998; 30: 152-7

[25] Woolf-May K, Kearney EM, Owen A, Jones DW, Davison RC, Bird SR. The efficacy of accumulated short bouts versus single daily bouts of brisk walking in improving aerobic fitness and blood lipid profiles. Health Educ Res 1999; 14: 803-15.

[26] Coyle EF. Very intense exercise-training is extremely potent and time efficient: a reminder. J Appl Physiol 2005; 98: 1983-4.

[27] Burgomaster KA, Hughes SC, Heigenhauser GJF, Bradwell SN, Gibala MJ. Six sessions of sprint interval training increases muscle oxidative potential and cycle endurance capacity in humans. J Appl Physiol 2005; 98: 1985-90.

[28] Gibala MJ, Little JP, van Essen M, et al. Short-term sprint interval versus traditional endurance training: similar initial adaptations in human skeletal muscle and exercise performance. J Physiol 2006; 575: 901-11.

[29] Howarth KR, Burgomaster KA, Phillips SM, Gibala MJ. Exercise training increases branched-chain oxoacid dehydrogenase kinase content in human skeletal muscle. Am J Physiol Regul Integr Comp Physiol 2007; 293: 1335.

[30] Getzen TE. Health economics: fundamentals and flow of funds. 2nd ed. New York: Wiley 2004.

[31] Steimetz SSC, Brownstone D. Estimating commuters'value of time" with noisy data: a multiple imputation approach. Transport Res Part B 2005; 39: 865-89.

[32] Noble BJ, Borg GA, Jacobs I, Ceci R, Kaiser P. A category-ratio perceived exertion scale: relationship to blood and muscle lactates and heart rate. Med Sci Sports Exerc 1983; 15: 523-8.

[33] Noble BJ, Robertson RJ. Perceived exertion. Human Kinetics: Champaign, IL 1996.

[34] Chapman GB, Elstein AS. Valuing the future: temporal discounting of health and money. Med Decis Making 1995; 15: 373-86.

[35] Frederick S, Loewenstein G, O'donoghue T. Time discounting and time preference: a critical review. J Econ Lit 2002; 40: 351-401.
[36] Ibbotson RG, Brinson GP. Global Investing: The Professional's Guide to the World Capital Markets. New York: McGraw-Hill 1993.

[37] Brannon I. What Is a life worth? Regulation 2004; 27: 60-3.

[38] Viscusi WK, Aldy JE. The value of a statistical life: a critical review of market estimates throughout the world. J Risk Uncertain 2003; 27: 5-76.

[39] De Blaeij A, Florax R, Rietveld P, Verhoef E. The value of statistical life in road safety: a meta-analysis. Accid Anal Prev 2003; 35: 973-86.

[40] Tversky A, Kahneman D. Advances in prospect theory: cumulative representation of uncertainty. J Risk Uncertain 1992; 5: 297-323.

[41] Alberini A, Cropper M, Krupnick A, Simon NB. Does the value of a statistical life vary with age and health status? Evidence from the US and Canada. J Environ Econ Manage 2004; 48: 769-92.

[42] Akerlof GA, Scarry S. Behavioral macroeconomics and macroeconomic behavior. In: Ackerlof G, Ed. Explorations in pragmatic economics. Oxford: Oxford University Press 2005.

[43] Pratt M, Macera CA, Blanton C. Levels of physical activity and inactivity in children and adults in the United States: current evidence and research issues. Med Sci Sports Exerc 1999; 31: 526-33.

[44] Malina RM. Tracking of physical activity and physical fitness across the lifespan. Res Q Exerc Sport 1996; 67: 48-57.

[45] Morrow JR, Jr, Krzewinski-Malone JA, Jackson AW, Bungum TJ, FitzGerald SJ. American adults' knowledge of exercise recommendations. Res Q Exerc Sport 2004; 75: 231-7.

[46] Blair SN, LaMonte MJ, Nichaman MZ. The evolution of physical activity recommendations: how much is enough? Am J Clin Nutr 2004; 79: 913-20.

[47] Activity A. Physical activity and public health: updated recommendation for adults from the American College of Sports Medicine and the American Heart Association. Med Sci Sports Exerc 2007; 39: 1423-34

[48] Sallis JF, Linton L, Kraft MK. The first Active Living Research Conference: growth of a transdisciplinary field. Am J Prev Med 2005; 28: 93-5.

[49] Everett MD, Anthony MS. Healthcare costs and benefits: a future direction. J Personal Finance 2005; 4: 69-85.

[50] Nelson ME, Rejeski WJ, Blair SN, et al. Physical activity and public health in older adults: recommendation from the American College of Sports Medicine and the American Heart Association. Circulation 2007; 116: 1094-105.

[51] Mittleman MA, Maclure M, Tofler GH, Sherwood JB, Goldberg RJ, Muller JE. Triggering of acute myocardial infarction by heavy physical exertion--protection against triggering by regular exertion. N Engl J Med 1993; 329: 1677-83.

[52] Williams PT. Physical fitness and activity as separate heart disease risk factors: a meta-analysis. Med Sci Sports Exerc 2001; 33: 75461 .

[53] Barnes DE, Yaffe K, Satariano WA, Tager IB. A longitudinal study of cardiorespiratory fitness and cognitive function in healthy older adults. J Am Geriatr Soc 2003; 51: 459-65.

[54] Bixby WR, Spalding TW, Haufler AJ, et al. The unique relation of physical activity to executive function in older men and women. Med Sci Sports Exerc 2007; 39: 1408-16.

[55] Whaley MH, Brubaker PH, Otto RM, Armstrong LE. American College of Sports Medicine, ACSM's Guidelines for Exercise Testing and Prescription. Philadelphia: Lippincott Williams \& Wilkins, 2006.

[56] Green PE, Srinivasan V. Conjoint analysis in marketing: new developments with implications for research and practice. J Market 1990; 54: 3-19.

[57] Ryan M, Farrar S. Using conjoint analysis to elicit preferences for health care. BMJ 2000; 320: 1530-3. 